\title{
Quantum Chemical Investigation of C12 and C6 Position of Oseltamivir Sialidase Antiviral Inhibitor
}

\author{
Krishnan Chandrasekaran \\ Science division, Department of Chemistry, Vel Tech University, Chennai 600 062, India.
}

\begin{tabular}{|c|c|}
\hline ARTICLE INFO & ABSTRACT \\
\hline Article history: & The ab initio and DFT investigation of $\mathrm{C} 12$ \& $\mathrm{C} 6$ position of oseltamivir sialidase inhibitor reveals that the \\
\hline Received on: 17/06/2015 & absence of pyranose oxygen ring in the inhibitor structure drastically increases binding affinity of the inhibitor in \\
\hline Revised on: 12/07/2015 & relation to the pyranose based inhibitors. The investigation further reveals that the methyl and ethyl group at the \\
\hline Accepted on: 17/07/2015 & C12 position have substantial binding affinity due to their inherent hyperconjugative and charge transfer effects \\
\hline Available online: $27 / 09 / 2015$ & between $\mathrm{C} 4$ and $\mathrm{C} 13$ bond. The analysis at $\mathrm{C} 6$ position of oseltamivir inhibitor discloses that the methyl amine \\
\hline $\begin{array}{l}\text { Key words: Oseltamivir, } \\
\text { Sialidase inhibitors. Binding } \\
\text { affinity, influenza virus. }\end{array}$ & $\begin{array}{l}\text { Hence, the investigation validates that the } 12 \text {-methyl-oseltamivir, 12-ethyl-oseltamivir and 6-methylamine- } \\
\text { oseltamivir inhibitor become the potential candidate for the development effective sialidase antiviral inhibitors. }\end{array}$ \\
\hline
\end{tabular}

\section{INTRODUCTION}

Influenza virus is a RNA virus which contains two surface proteins namely hemaglutinnin (HA) and neuraminidase (Chak et al., 2007). Among the two proteins, hemagglutinin is the prime target of vaccines and neuraminidase is the main target of anti viral drugs. Vaccines against influenza virus are frequently inactive due to the rapid emergence of mutant viral antigens (Gerdon et al., 2005) and hence, it creates an inevitable need of antiviral drugs. NA is an enzyme protein, the influenza virus cleaves the alpla ketosidic linkage of the neuraminidase active site (sialic acid) and the adjacent arginine amino residue of the host cell receptor (Jarreau et al., 1992) The cleavage process proliferate the viral infection to the other cells and thus continues its lifecycle. Inhibiting the activity of neuraminidase will trap the virus particle inside the infected host cell. The binding site of NA is smaller and has a highly selective binding pocket and hence, this site is an ideal target for design of antiviral drugs against a broad range of influenza virus (Vincent et al., 2003). The first two antiviral drugs amantadine and rimantadine which block the M2 protein ion channel function will be effective in blocking

\footnotetext{
* Corresponding Author

Chandrasekaran Krishnan, Science division, Chemistry department, Vel Tech University, Chennai, India.Email: nuskrishnan@yahoo.com.sg
}

only the influenza A type virus (Hayden et al., 1992) and fails to contain the influenza B type virus, moreover resistant mutants are rapidly generated for the above compounds. Therefore alternative treatment to contain the influenza virus focused towards the development of sialidase antiviral inhibitors. Meanwhile, antiviral inhibitor treatments for influenza virus (neuraminidase inhibitors) have been emerged as potential therapeutics to treat the influenza. The two such compounds developed are zanamivir (Macdonald et al., 2005) and oseltamivir (Harrington et al., 1996) successfully contains the rapid generation of mutants of influenza A \& B. The developments of zanamivir, oseltamivir, and BCX (Chand et al., 2004) antiviral inhibitors are purley based on the transition structure of neuraminidase with its natural substrate sialic acid. Sialic acid is the active site of neuraminidase and it has the five binding pocket; each of pocket bound to the adjacent amino acid residues, its structure is shown in the figure-1.

Natural substrate sialic acid cleaves from the neuraminidase protein by enzyme catalytic process and proliferates the viral infection due to the low binding affinity with its receptor protein residues. The process of cleavage of sialic acid from the adjacent arginine amino acid residue proceeds through the formation of sialyl cation intermediate (Colmann et al., 1994) therefore the search for neuraminidase inhibitor is focussed on the structure of cation intermediate. 
The first developed sialcidase inhibitor is DANA (Alexander et al., 2000). The DANA is the dehydrated derivative of the sialic acid; which resembles the transition state geometry of the sialyl cation intermediate formed during the sialidase catalytic process. The replacement of hydroxyl group in DANA with amino group resulted in the 4-amino-DANA; which is more potent than DANA. All the inhibitors DANA, 4-amino-DANA, 4guanidino-DANA contains oxygen atom in the ring and thereby it suffers in conformation flexibility during binding with receptors. Tamiflu or oseltamivir (Lew et al., 2000) is the first inhibitor developed with carbocyclic ring which does not have oxygen atom in the ring with the high degree of stability. Moreover this nonpyranose inhibitor reduces the polarity of the compound and increases the bio-availability of the compound. Hence, this carbocyclic inhibitor established a new era in anti influenza drugs. It appears that $\mathrm{C} 7$ and $\mathrm{C} 12$ position of tamiflu remains free without involving in any interaction with its receptor amino acid residues. Hence, the investigation of $\mathrm{C} 7$ and $\mathrm{C} 12$ position of oseltamivir with various substituent could improve its potency and will acts as potential antiviral inhibitor. Besides, recent ab initio investigation (Krishnan et al., 2015) of DANA based sialidase antiviral inhibitors reveals that substituent at certain position drastically increases the binding affinity of the inhibitor and becomes the new antiviral drugs.

Fig. 1: Structure of sialic with Five Binding Pockets.

\section{COMPUTATIONAL METHOD}

All the oseltamivir compounds are invariably optimized using the HF/6-31G(d) level of theory. The oseltamivir compounds and its substituted compounds are optimized in anionic form using the above level of theory. Single point energy calculation of all the optimized oseltamivir compounds were carried out using B3LYP/6-31G(d) level of theory. Single point energy calculations of all the compounds were performed in both gas phase and solvent phase. CPCM model [Conductor like polarized continuum model] is employed for the solvent energy calculation and water is the only solvent used for solvation models. Likewise the receptor model compound guanidino cation compound is optimized using the HF/6-31G(d) level of theory and single point energy calculation is performed using B3LYP/6$31 \mathrm{G}(\mathrm{d})$ level of theory in both gas phase and solvent phase (CPCM solvent model). The binding affinity of oseltamivir and its substituted compounds were estimated by using the following equation,

$$
\Delta \mathrm{E}_{(\text {Bind })}=\mathrm{E}_{\mathrm{g}(\text { Substrate-Ligand Complex })}-\left(\mathrm{E}_{(\text {substrate })}+\mathrm{E}_{(\text {Ligand }) \mathrm{g}}\right)
$$

\section{RESULTS AND DISCUSSION}

Oseltamivir on interaction with methyl guanidino provides ligand binding energy of $122.2 \mathrm{kca} / \mathrm{mol}$. Methyl guanidino is used as model compound to replace the amino acid residue (argnine) as a receptor binding with the oseltamivir. The structure of oseltamivir is shown in fugure 2. But the pyranose derivative yields binding energy of $109.65 \mathrm{kcal} / \mathrm{mol}$. So it is apparent that the non-pyranose derivative acts as the powerful inhibitors. Substitution of methoxy group at the $\mathrm{C} 12$ position provides the binding affinity of $121.56 \mathrm{kcal} / \mathrm{mol}$; so it shares same platform with with oseltamivir inhibitor. The introduction of ethoxy group at the $\mathrm{C} 12$ position does not produce significant effect on the binding affinity due to the highly polar nature. It provides the binding energy of $121.66 \mathrm{kcal} / \mathrm{mol}$. The polar nature of methoxy and ethoxy group decreases the negative inductive effect between conjugative carbon $\mathrm{C} 4=\mathrm{C} 13$ bond and thus have little effect on binding affinity.

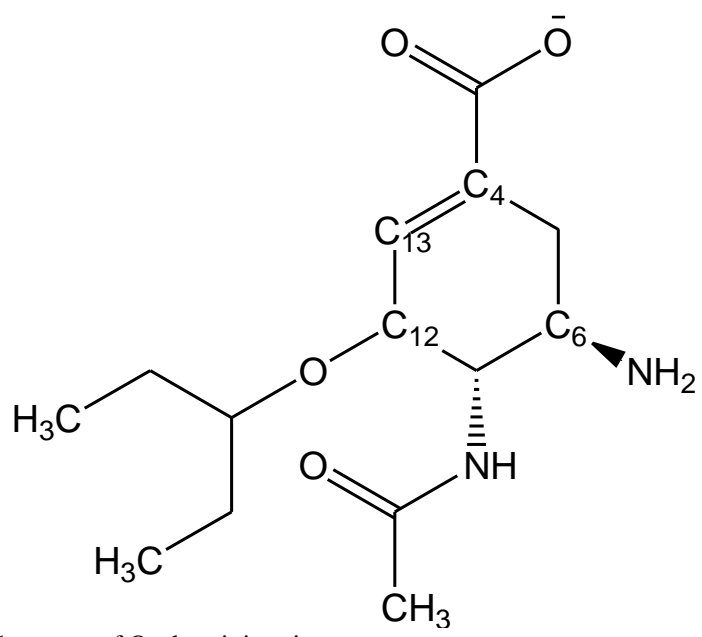

Fig. 2: Structure of Oseltamivir anion.

The introduction of methyl group at the $\mathrm{C} 12$ position of oseltamivir increases the binding affinity to $120.04 \mathrm{kcal} / \mathrm{mol}$ and it is higher than the parent oseltamivir inhibitor. Increase in binding affinity is attributed to the fact the presence of methyl group promotes the hyperconjugative effect and pi electron delocalization between $\mathrm{C} 13$ and $\mathrm{C} 4$ carbon atoms. In addition to the hyperconjugative effect, the absence of polar group also accounts for the higher binding energy of 12-methyl oseltamivir inhibitor. 
Table 1 : Effect of $\mathrm{C} 12$ substituents on the binding affinity of oseltamivir.
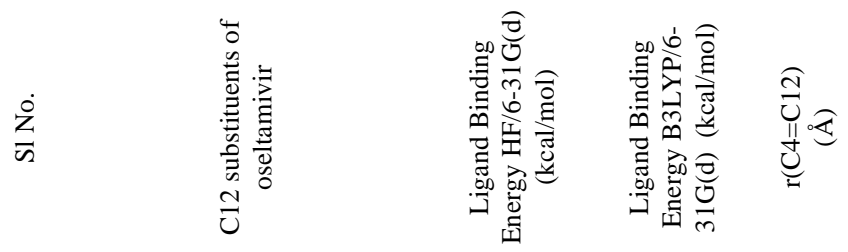

\begin{tabular}{llccc}
\hline 1 & Oseltamivir & 118.34 & 122.21 & 1.326 \\
2 & Methoxy & 117.27 & 121.56 & 1.327 \\
3 & Ethoxy & 117.34 & 121.66 & 1.327 \\
4 & Methyl & 119.91 & 124.03 & 1.327 \\
5 & Ethyl & 119.87 & 124.12 & 1.326 \\
6 & Chlorine & 110.9 & 117.72 & 1.326 \\
7 & Fluorine & 116.67 & 121.21 & 1.326 \\
8 & Thiol & 115.38 & 119.32 & 1.326 \\
9 & Guanidino & 115.67 & 118.38 & 1.327 \\
10 & Tri fluoro carbon & 115.53 & 120.81 & 1.324 \\
\hline
\end{tabular}

Substitution of ethyl group at the $\mathrm{C} 12$ position increases the binding affinity to $124.03 \mathrm{kcal} / \mathrm{mol}$. However, in relative to the methyl group; it produces the same effect in binding affinity and which implies that the lengthening of alkyl group does not increase the binding affinity. It is apparent from table 1 that the introduction of chlorine and fluorine at the $\mathrm{C} 12$ position decreases the binding affinity to 117.72 and $121.20 \mathrm{kcal} / \mathrm{mol}$. It appears that both the halogens have failed to form strong hydrogen bonds with receptor and thus attains moderate binding affinity. Substitution of thiol at the $\mathrm{C} 12$ does not produce the required effect and it attains the binding energy of $119.32 \mathrm{kcal} / \mathrm{mol}$.

Likewise the substitution of guanidino and tri fluoro carbon decreases the binding affinity of oseltamivir due to the lack of delocalization of $\pi$ electrons between $\mathrm{C} 2=\mathrm{C} 14$ bond. It is clear from table 1 that the substituents like tri fluoro cadrbon, guanidino and thiol at the $\mathrm{C} 12$ position decreases the electron density on $\mathrm{C} 2$ atom and decreases the binding affinity. Summary of table 1 predicts that the alkyl groups like ethyl and methyl increases the binding affinity of oseltamivir and become the potent antiviral inhibitor.

\section{SOLVATED LIGAND BINDING ENERGY}

The study of binding affinity of tamiflu in solvent phase in inevitable because its interaction with protein and amino acids are involved in the solvent phase. Parent oseltamivir provides provides solvated binding energy of $20.82 \mathrm{kcal} / \mathrm{mol}$. Substitution of methoxy group at the $\mathrm{C} 12$ position decreases the solvated binding energy to $14.03 \mathrm{kcal} / \mathrm{mol}$.

The hydrophobic methoxy group fails to bind effectively with receptor and as a result it attains low binding affinity. Introduction of ethoxy group at the $\mathrm{C} 12$ position also decreases the binding affinity, it is due to the polarisation of the ethoxy group at the $\mathrm{C} 12$ position. Likewise methyl group at $\mathrm{C} 12$ position decreases the binding affinity to $13.84 \mathrm{kcal} / \mathrm{mol}$ due to its bulkiness and hydrophobic nature. Substitution of ethyl at C12 position decreases the binding affinity to $14.54 \mathrm{kcal} / \mathrm{mol}$. It is clear from table 2 that alkyl group could not increase the binding affinity in solvent due its bulky hydrophobic behaviour. However, alkyl group increases the binding affinity in gas phase and becomes the potential candidate for the antiviral inhibitor. Substitution of chlorine in the $\mathrm{C} 12$ position decreases the binding affinity and yields binding energy of $117.72 \mathrm{kcal} / \mathrm{mol}$. Likewise fluorine at C12 position of oseltamivir has no effect on binding affinity and attains binding energy of $121.2 \mathrm{kcal} / \mathrm{mol}$

Table 2: Solvated Binding energy of C12 substituents .

\begin{tabular}{|c|c|c|c|c|}
\hline$\frac{\dot{\theta}}{\tilde{r}}$ & 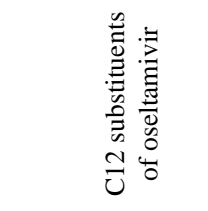 & 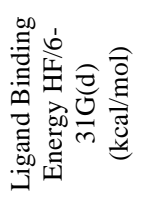 & 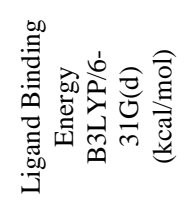 & 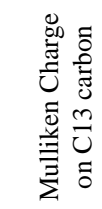 \\
\hline 1 & Oseltamivir & 3.59 & 20.83 & -0.107 \\
\hline 2 & Methoxy & 6.66 & 14.81 & -0.1 \\
\hline 3 & Ethoxy & 6.85 & 15.07 & -0.101 \\
\hline 4 & Methyl & 5.11 & 13.84 & -0.112 \\
\hline 5 & Ethyl & 5.36 & 14.54 & -0.112 \\
\hline 6 & Chlorine & 0.63 & 12.85 & -0.093 \\
\hline 7 & Fluorine & 5.86 & 14.12 & -0.093 \\
\hline 8 & Thiol & 5.38 & 13.69 & -0.1 \\
\hline 9 & Guanidino & 11.19 & 16.66 & -0.096 \\
\hline 10 & Tri fluoro carbon & 5.18 & 14.09 & -0.093 \\
\hline
\end{tabular}

It is clear from table 2 that halogens at the $\mathrm{C} 12$ position disperses the electron density at the $\mathrm{C} 2$ carbon and causes low binding affinity and hence its effect remains futile. Substitution of sulphur based thiol at the $\mathrm{C} 12$ position also decreases the binding affinity and attains the binding energy of $119.32 \mathrm{kcal} / \mathrm{mol}$. Substitution of thiol decreases the binding affinity due to hydrophobic nature. Substitution of guanidino at the $\mathrm{C} 12$ position decreases the binding affinity to $118.38 \mathrm{kcal} / \mathrm{mol}$.

Guanidino substituent decreases the electrostatic and polarization charge transfer in the solvation medium and thus decreases the binding affinity. Trifluoro carbon at the $\mathrm{C} 12$ position have little effect on the binding affinity and attains the binding energy of $120.8 \mathrm{kcal} / \mathrm{mol}$. Tri fluoro carbon at $\mathrm{C} 12$ position increases the charge transfer between $\mathrm{C} 12=\mathrm{C} 3$ bond by decreasing the bond length from $1.326 \AA$ to $1.324 \AA$. Although it has effective charge transfer between $\mathrm{C} 12$ and $\mathrm{C} 3$ carbon; the hydrophobic nature of fluorine causes low binding affinity.

\section{EFFECT OF C6 SUBSTITUENT ON OSELTAMIVIR}

The di-methoxy group present at the C12 position of oseltamivir kept constant and the amino group present at the C6 position replaced by various substituents to evaluate the binding affinity of C6 modified oseltamivir and reported results in table 3. Introduction of guanidino at the $\mathrm{C} 6$ position provides binding energy of $121.35 \mathrm{kcal} / \mathrm{mol}$ and thereby it shares same platform with amino group. The presence of two amino groups in the guanidino substituent does not make any significant change in binding affinity. Substitution of methyl group at C6 position of oseltamivir yields binding energy of $121.15 \mathrm{kcal} / \mathrm{mol}$. It appears that the methyl group at C6 also has the same effect as amino group. 
Table 3: Effect of C6 Substituents on the Binding Affinity of oseltamivir Compound.

\begin{tabular}{|c|c|c|c|c|c|}
\hline 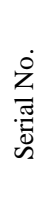 & 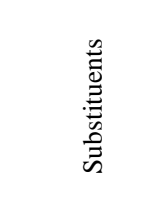 & 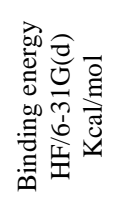 & 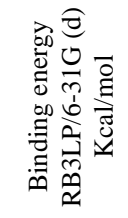 & 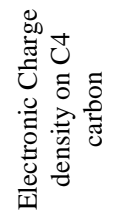 & 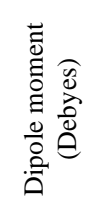 \\
\hline 1 & Oseltamivir & 118.34 & 122.21 & -0.107 & 9.07 \\
\hline 2 & Guanidino & 117.73 & 121.35 & -0.119 & 11.61 \\
\hline 3 & Methyl & 116.82 & 121.15 & -0.092 & 10.7 \\
\hline 4 & Fluorine & 114.55 & 119.45 & -0.12 & 11.39 \\
\hline 5 & Methyl amine & 117.06 & 121.36 & -0.092 & 10.61 \\
\hline 6 & Thiol & 115.07 & 118.95 & -0.091 & 10.96 \\
\hline
\end{tabular}

It is apparent from table 3 that the methyl group increases the electron charge density on $\mathrm{C} 2$ by hyperconjugative effect and thus increases the binding affinity even though its bulkiness. Introduction of fluorine at the $\mathrm{C} 6$ position provides the binding affinity of $119.45 \mathrm{kcal} / \mathrm{mol}$. It exhibits only a minor decrease in binding affinity in relative to the oseltamivir due to its negative inductive effect. The introduction of methyl amine at the C6 position attains the binding energy of $121.36 \mathrm{kcal} / \mathrm{mol}$. It is clear from table 3 that the methyl amine increases the binding affinity by conjugative inductive effect and provides better binding affinity. The presence of amine group in methyl amine offers greater hydrogen bonding ability with the neighboring receptors and increases the binding affinity. Introduction of thiol at the C6 position decreases the binding affinity due to lack of hydrogen bonding with the receptors. The substitution of thiol at the C6 position poloarizes the oseltamivir molecule and decreases the binding affinity. In a nutshell the investigation reveals that among the five substituents only methylamine at C6 position increases the binding affinity and therefore it could acts a potential anti viral sialidase inhibitor in the future antiviral drug era.

\section{CONCLUSION}

$\mathrm{Ab}$ initio and DFT investigation of $\mathrm{C} 12$ position of sialidase oseltamivir inhibitor reveals thatthe methyl and ethyl group have attained higher binding affinity due to their hyper conjugative effects and charge transfer effects. 12-methyl oseltamivir and 12-ethyl oseltamivir could become the potential candidate for the development of potent sialidase antiviral inhibitors. Apart from the alkyl group, the alkoxy groups such as methoxy and ethoxy group also shows better binding affinity than the parent oseltamivir compound and further investigation on this compounds might produce promising results to produce the more potent sialidase inhibitors. The analysis of binding affinity of $\mathrm{C} 12$ substituents of oseltamivir compound in solvent phase indicates that the parent oseltamivir attains better binding affinity that all other substituents due to the lack of polarization effect and hydrogen bonding affinity with the neighboring receptors. The theoretical investigation of C6 position of oseltamivir inhibitor discloses that among all the five substituent, the methyl group increases the binding affinity. The presence of methyl group at the
C6 position increases the charge transfer between $\mathrm{C} 4=\mathrm{C} 13$ by hyperconjugative effect and consequently increases the binding affinity. Hence the investigation concludes that the 6-methyl oseltamivir and 12-methyl oseltamivir compounds have shown better binding affinity and thus it paves way for development and design of potential sialidase antiviral inhibitor.

\section{REFERENCES}

Alexander DJ. A review of avian influenza in different bird species. Vet. Microbiol., 2000; 74(1-2): 3-9.

Chak S, and Supa H. Structural Information and Computational Methods Used in Design of Neuraminidase Inhibitors. Current ComputerAided Drug Design, 2007; 3: 113-132.

Chand P, Babu YS, Bantia S, Rowland S, Dehghani A, Kotian PL, Hutchison TL, Ali S, Brouillette W, El-Kattan Y, Lin TH. Syntheses and Neuraminidase Inhibitory Activity of Multisubstituted Cyclopentane Amide Derivatives J.Med. Chem., 2004; 47: 1919-1924.

Colmanm, PM, Influenza virus neuraminidase: Structure, antibodies and inhibitors. Protein Science. 1994; 3: 1687-1696.

Cramer, CJ Truhlar, DG, Implicit Solvation Models: Equilibria, Structure, Spectra, and Dynamics. Chem Rev., 1999; 99; 2161-2190.

Gerdon AE, Wright DW, Cliffel DE, Hemagglutinin Linear Epitope Presentation on Monolayer-Protected Clusters Elicits Strong Antibody Binding. Bio-macromolecules, 2005; 6: 3419-3424.

Hayden FG, Couch RB, Clinical and epidemiological importance of influenza A viruses resistant to amantadine and rimantadine. Rev. Med. Virol., 1992; 2: 89-96.

Harrington PJ, Brown JD, Foderaro T, Hughes RC. The Structures of Salmonella typhimuriumLT2 Neuraminidase and its Complexes with Three Inhibitors at High Resolution, J. Mol. Biol., 1996; 259: 264-280.

Jarreau PH, Harf A, Levame M, Lambre CR, Lorino $\mathrm{H}$. Macquin- Mavier I. Effects of Neuraminidase on Airway Reactivity in the Guinea Pig, Am. Rev. Respir. Dis., 1992; 145: 906-912.

Krishnan C. $A b$ initio and DFT investigation of C4 \& C7 position of sialidase antiviral inhibitor. J. Chem. Pharm. Res., 2015; 7(4): 425-430

Lew W. Chen X, Kim CU, Discovery and Development of GS 4104 (oseltamivir) An Orally Active Influenza Neuraminidase Inhibitor. Curr Med Chem, 2000; 7(6): 663-668.

Macdonald SJF. Cameron R, Demaine DA, Fenton RJ, Foster G, Gower D, Hamblin JN, Hamilton S, Hart GJ, Hill A.P, Inglis GGA, Jin B, Jones HT, McConnell DB, McKimm-Breschkin J, Mills OG, Nguyen V, Owens IJ, Parry N, Shanahan SE, Smith D, Watson KG, Wu WY, Tucker SP. Dimeric Zanamivir Conjugates with various linking groups are potent, Long-Lasting Inhibitors of Influenza Neuraminidase Including H5N1 Avian Influenza. J. Med. Chem, 2005; 48: 2964-2971.

Vincent S, Kent D, Stewart CJ, Maring SM, Vincent G, Yugui G, Gary W, Yuanwei C, Minghua S, Chen Z, April L, Kennedy DL, Madigan YX, Ayda S, Warren , Graeme L, Thomas S, Hing LS, Jonathan G, Dale K. Influenza Neuraminidase Inhibitors: Structure-Based Design of a Novel Inhibitor Series. Biochemistry. 2003; 42: 718-722.

\section{How to cite this article:}

Chandrasekaran Krishnan. Quantum Chemical Investigation of C12 And C6 Position of Oseltamavir Sialidase Antiviral Inhibitor. J App Pharm Sci, 2015; 5 (09): 120-123. 